\title{
A Dynamic Committee Scheme on Multiple-Criteria Linear Programming Classification Method
}

\author{
Meihong $\mathrm{Zhu}^{1,2}$,Yong $\mathrm{Shi}^{1}$, Aihua $\mathrm{Li}^{1}$, and Jing $\mathrm{He}^{1}$ \\ ${ }^{1}$ CAS Research Center on Data Technology and Knowledge Economy \\ Management School, Graduate University of CAS, \\ Beijing 100080, China \\ ${ }^{2}$ Statistics School, Capital University of Economics and Business \\ Beijing100070, China \\ \{zhumh,yshi, lah04b, jinghe\}@mails.gucas.ac.cn
}

\begin{abstract}
This paper aims to provide a scheme for effectively and efficiently finding an approximately optimal example size with respect to a given dataset when using Multiple-Criteria Linear Programming (MCLP) classification method. By integrating techniques of both progressive sampling and classification committee, it designs a dynamic classification committee scheme for MCLP. The experimental results have shown that our idea is feasible and the scheme is effective and efficient for exploring an approximately optimal sample size. The empirical results also help us to further investigate some general specialties of MCLP, such as the more general function expressions reflecting the relationship between accuracy and sample size, and between computing cost and sample size.
\end{abstract}

Keywords: Classification, Multiple-Criteria Linear Programming, Progressive Sampling, and Committee.

\section{Introduction}

In data mining field, Multiple-Criteria Linear Programming (MCLP) classification method is an outstanding classification tool [1-5]. But, just like many other classification tools, its computation efficiency is sometimes low when faced with large and high-dimension datasets. Among research efforts to improve classification efficiency of MCLP, sampling is an important approach. In practice, MCLP is often executed on a one-off (static) selected sample, while the sample size is often determined by analysts subjectively. The subsequential problem is that we can't judge whether the one-off selected sample is good enough for analyses. If the sample is small, it can't reflect the original data sufficiently; contrarily, if it is too large, computation cost will be unacceptable. So, how to identify an appropriate sample size is the key to the success of applying sampling techniques to MCLP. Among various sampling approaches, progressive sampling is a well-known one for finding an approximately optimal example size $[6,7]$. 
This paper designs a scheme to effectively and efficiently find an approximately optimal example size for MCLP classification method with respect to a given dataset. This scheme integrates techniques of both progressive sampling and classification committee. First, a new non-standard arithmetic progressive sampling procedure is implemented to determine example size of each step. Secondly, at each step of progressive sampling, a classification committee [8-13] is constituted by 9 samples with the same size. Finally, by comparing the accuracies of adjacent committees dynamically and interactively, an approximately optimal example size for MCLP is found. Resumptively, this scheme can be called a Dynamic Committee Scheme on MCLP classification method.

To illuminate our idea simply, this paper only focuses on two-group classification problems. Two real-life databases with different sizes are applied to verify our idea. Experiment results provide us following conclusions: Firstly, the Dynamic Committee Scheme on MCLP can effectively find the approximately optimal example size for MCLP classification method with respect to a given dataset, i.e., the approximately optimal example size really exists. Secondly, it can efficiently find the approximately optimal results, i.e., the computation is acceptable. Additionally, these experiment results help us to execute an in-depth study of specialties of MCLP, such as the relationship between classification accuracy and sample size, and that between computing cost and sample size, and that between computing cost and number of attributes.

The rest of the paper is organized as follows. Section 2 introduces some background knowledge for latter analyses. Section 3 describes our dynamic Classification Committee scheme on Multiple-Criteria Linear Programming classification method in detail. Section 4 introduces experimental operations and analyzes corresponding results. The last section concludes our work and presents future research directions.

\section{Background Knowledge}

To understand our idea and method, three aspects of background knowledge are needed.

\subsection{Two-Class MCLP Classification Model}

MCLP classification model is first presented by Shi et al.[1]. A two-class MCLP model can be depicted as follows:

Given a training dataset which has $p$ predictive variables (attributes) $a=\left(a_{l}, \ldots\right.$, $\left.a_{p}\right)$ and $n$ cases, let $A_{i}=\left(A_{i l}, \ldots, A_{i p}\right)$ be the data for the variables in ith case, where $i$ $=1, \ldots, n$. We hope to explore the best coefficients of $p$ variables denoted by $X=$ $\left(x_{1}, \ldots, x_{p}\right)^{T}$, and a boundary value $b$ (a scalar ) to separate existing data into two classes: $G_{l}$ and $G_{2}$; that is,

$$
A_{i} X \leq b, \forall A_{i} \in G_{1} \text { and } A_{i} X \geq b, \forall A_{i} \in G_{2}
$$


In this expression, $A_{i} X$ is a linear function of attribute variables $X$ used for classifying each labeled or unlabeled case. To measure the separation degree of $G_{1}$ and $G_{2}$, we define:

$$
\begin{aligned}
& \alpha_{\mathrm{i}}=\text { the overlapping degree of two classes boundary for case } A_{i} \\
& \beta_{i}=\text { the distance of case } A_{i} \text { from its adjusted boundary. }
\end{aligned}
$$

The objective function is minimizing the sum of $\alpha_{i}$ and maximizing the sum of $\beta_{i}$ simultaneously. So there is following classification model (M1).

$$
\begin{aligned}
& \text { Minimize } \sum_{i} \alpha_{i} \text { and Maximize } \sum_{i} \beta_{i} \\
& \text { Subject to: } \\
& A_{i} X=b+\alpha_{i}-\beta_{i}, \forall A i \in G_{1}, \\
& A_{i} X=b-\alpha_{i}+\beta_{i}, \forall A i \in G_{2} .
\end{aligned}
$$

Where $A_{i}$ are given; $X$ and $b$ are unrestricted; and $\alpha_{i}$ and $\beta_{i} \geq 0$.

This multi-objectives linear programming problem can be transformed into a single-objective model by introducing the compromise solution approach [2,3].We assume the "ideal value" of $-\Sigma_{i} \alpha_{i}$ be $\alpha^{*}>0$ and the "ideal value" of $\Sigma_{i} \beta_{i}$ be $\beta^{*}>0$. If $-\sum_{i} \alpha_{i}>\alpha^{*}$, we define the regret measure as $-d_{\alpha}^{+}=\Sigma_{i} \alpha_{i}+\alpha^{*}$; otherwise, it is 0 . If $-\Sigma_{i}$ $\alpha_{i}<\alpha^{*}$, the regret measure is defined as $d_{\alpha}{ }^{-}=\alpha^{*}+\Sigma_{i} \alpha_{i}$; otherwise, it is 0 . Thus, we have (i) $\alpha^{*}+\sum_{i} \alpha_{i}=d_{\alpha}^{-}-d_{\alpha}^{+}$, (ii) $\left|\alpha^{*}+\sum_{i} \alpha_{i}\right|=d_{\alpha}^{-}+d_{\alpha}^{+}$, and (iii) $d_{\alpha}^{-}, d_{\alpha}^{+} \geq$ 0. Similarly, we have $\beta^{*}-\Sigma_{i} \beta_{i}=d_{\beta_{-}}-d_{\beta}{ }^{+},\left|\beta^{*}-\Sigma_{i} \beta_{i}\right|=d_{\beta}{ }^{-}+d_{\beta}{ }^{+}$, and $d_{\beta}{ }^{-}, d_{\beta}{ }^{+} \geq 0$. So M1 can be changed into M2.

$$
\begin{aligned}
& \text { Minimize } d_{\alpha}^{-}+d_{\alpha}^{+}+d_{\beta}^{-}+d_{\beta}^{-} \\
& \text {Subject to: } \\
& \alpha^{*}+\sum_{i} \alpha_{i}=d_{\alpha}^{-}-d_{\alpha}^{+} \\
& \beta^{*}-\sum_{i} \beta_{i}=d_{\beta}^{-}-d_{\beta}^{+} \\
& A_{i} X=b+\alpha_{i}-\beta_{i}, \forall A i \in G_{1}, \\
& A_{i} X=b-\alpha_{i}+\beta_{i}, \forall A i \in G_{2} .
\end{aligned}
$$

Where $A_{i}, \alpha^{*}$, and $\beta^{*}$ are given, $X$ and $b$ are unrestricted, and $\alpha_{i}, \beta_{i}, d_{\alpha}{ }^{-}, d_{\alpha}{ }^{+}, d_{\beta}{ }^{-}, d_{\beta}^{+}$ $\geq 0$. If $b$ is given and $X$ is found, we can classify a labeled or unlabeled by using linear discriminant $A X$.

The standard two-class MCLP algorithm is based on the M2. It uses the idea of linear programming to determine a boundary separating classes. Comparing with other classification tools, it's simple and direct, free of the statistical assumptions, flexible in defining and modifying parameters, and high on classification accuracy [5]. It has been widely used in business fields, such as credit card analysis, fraud detecting, and so on. But faced with high dimension data or too much size of data, sometimes its computing efficiency is low.

\subsection{Progressive Sampling}

Progressive sampling [7] is a famous dynamic sampling method that can be used in large datasets. It attempts to maximize model accuracy as efficiently as possible. It is based on the fact that when the sample size is large enough, with the further increase 
of sample size, the model accuracy doesn't increase significantly. It is in fact a tradeoff between classification accuracy and computing cost. Because of dynamic interactions of sampling process, it overcomes the weakness of one-off (static) sampling.

It starts with a small sample $n_{0}$ and augment sample amount progressively at each step. At each step, a model is built from the current sample and is evaluated. If the resulting model has not reached user-specified accuracy threshold, the algorithm must operate once again. Generally, the relation between accuracy and corresponding sample size can be expressed in learning curve (Figure 1). The convergence $n_{\min }$ in the curve is the approximately optimal sample size.

In progressive sampling, two key aspects affecting sampling effectivity and efficiency are increasing mechanism of sample size and stopping or convergence criterion of sampling .

As for the first aspect, [6] proposed an arithmetic increasing schedule as

$$
S_{a}=\left\{n_{0}, n_{1}, n_{2}, \ldots, n_{k}\right\}=\left\{n_{0}, n_{0}+n_{\delta}, n_{0}+2 n_{\delta}, \ldots, n_{0}+k \cdot n_{\delta}\right\} .
$$

[7] presented a geometrical increasing schedule as

$$
S_{g}=\left\{n_{0}, n_{1}, n_{2}, \ldots, n_{k}\right\}=\left\{n_{0}, a \cdot n_{0}, a^{2} \cdot n_{0}, \ldots, a^{k} \cdot n_{0}\right\} .
$$

[6] drew a conclusion that arithmetic sampling is more efficient that one-off sampling, and [7] verified geometrical sampling is more efficient than arithmetic sampling.

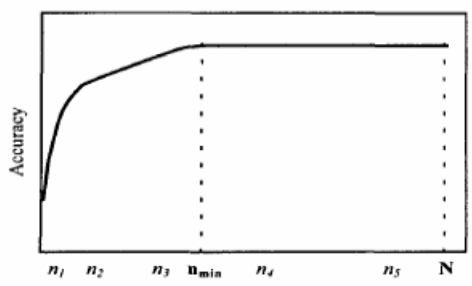

Fig. 1. Accuracy vs. sample size

\subsection{Classification Committee}

To improve prediction performance of an individual classifier, classification committee techniques are often used in many classification methods. There are many researches on committee/multi classifier/ensemble. The most popular guidelines for combining all individual classifiers are Bagging[8], Boosting[9],Random Subspace[10], and Random Forest[11]. There have been many variations of the above guidelines. The combination rules mainly include simple majority (mainly for Bagging), weighted majority (mainly for Boosting), minimum, maximum, product, average, Naive Bayes and so on[12].

Bagging is based on bootstrapping [13] and aggregating concepts, so it incorporates the benefits of both approaches. In Bagging, $m$ random independent bootstrap samples with the same size $n(\leq N)$ are taken out from the original dataset $N$, and then $m$ individual classifiers are developed, and finally a classification committee 
is made by aggregating these $m$ member classifiers using a simple majority vote. Bagging can be executed in parallel way, thus it is efficient.

\section{A Dynamic Committee Scheme on MCLP Classification Method}

In this paper, we want to explore the approximately optimal sample size $n_{\min }$ for MCLP classification method with respect to a given dataset. For simplicity, our research only focuses on two-class problems.

In our scheme, a committee is integrated by $m$ individual classifiers. The combination mechanism is a variation of Bagging technique, which takes out samples in a non-repetition way from original dataset $\mathrm{N}$ with $n_{i}<<N$.

To explain our method expediently, we define:

$\operatorname{acc}\left(n_{i}\right)=$ classification accuracy on test set computed by the committee with sample size $n_{i}$;

$c=$ user-defined accuracy threshold or criterion for stopping sampling.

To explore $n_{\text {min }}$, we design a progressive sampling scheme. In this scheme, sample size is increased according to a non-standard arithmetic progressive manner. Initially, sample sizes are arithmetically increased with a fixed increasing extent $n_{\delta}$ (see formula (4). When formula (5) is satisfied, the subsequent three sample sizes are increased according to a less degree $n_{\delta}^{\prime}$ as shown in formula (6).

$$
\begin{gathered}
\left\{n_{0}, n_{1}, n_{2}, \ldots, n_{k}\right\}=\left\{n_{0}, n_{0}+n_{\delta}, n_{0}+2 n_{\delta}, \ldots, n_{0}+k \cdot n_{\delta}\right\} \\
\operatorname{acc}\left(n_{k-1}\right)-\operatorname{acc}\left(n_{k-2}\right) \leq c, \text { and } \operatorname{acc}\left(n_{k}\right)-\operatorname{acc}\left(n_{k-1}\right) \leq c \\
\left\{n_{k+1}, n_{k+2}, n_{k+3}\right\}=\left\{n_{k}+n_{\delta}^{\prime}, n_{k}+2 n^{\prime}{ }_{\delta}, n_{k}+3 n_{\delta}^{\prime}\right\}, \text { where } n_{\delta}^{\prime}<n_{\delta}
\end{gathered}
$$

If formula (7) is satisfied, sampling process is cutoff; or else, later sample sizes are still increased according to the increment $n_{\delta}$.

$$
\operatorname{acc}\left(n_{k+1}\right)-\operatorname{acc}\left(n_{k}\right) \leq c, \operatorname{acc}\left(n_{k+2}\right)-\operatorname{acc}\left(n_{k+1}\right) \leq c \text {, and } \operatorname{acc}\left(n_{k+3}\right)-\operatorname{acc}\left(n_{k+2}\right) \leq c \text { (7) }
$$

When sampling process is stopped, the committee with the highest accuracy among the six sequential committees generated from the above six sample sizes $\left\{n_{k-2}, n_{k-1}, n_{k}, n_{k+1}, n_{k+2}, n_{k+3}\right\}$ is the final committee for prediction.

\section{Empirical Research}

To indicate effectivity and efficiency of our design, two real databases with different sizes are used in our experiment research. They are described as follows:

Table 1. Description of the Two Databases

\begin{tabular}{cccc}
\hline Database & number of predictive attributes & Size of training set & Size of test set \\
\hline Credit Card & 63 & $4000($ small $)$ & 1000 \\
\hline Census Income & 43 & $50000($ large $)$ & 20000 \\
\hline
\end{tabular}


In our design, we stipulate parameter $b=1, m=9$, and $c=0.3 \% \sim 0.5 \%$.

The operating results and intuitionistic expressions for two databases are shown in following tables and figures. Detailedly, "time" in following tables indicates average time for generating a single member classifier, and "accl" means accuracy rate for class 1 (majority class), and "acc2" means accuracy rate for class 2 (minority class), and "acc" denotes overall weighted-average accuracy.

\subsection{Experiments and Results}

As for the credit card dataset, considering its high dimension, we judge the computing cost is high, so parameter $c$ should be set comparatively high, and $n_{\delta}$ and $n_{0}$ comparatively low. Here $c$ is predefined as $0.3 \%, n_{0}$ as 200 , and $n_{\delta}$ as 200 . The initial scheme is $S=\{200,400,600,800\}$. The results show that the absolute improvement of acc on $n_{2}$ vs. $n_{1}, n_{3}$ vs. $n_{2}$ are all not more than $0.3 \%$, i.e., the results on different sample sizes are stabile, so latter sample sizes are changed at a less scope by $n_{\delta}^{\prime}=100$ sequentially. Then $\mathrm{S}=\{200,400,600,800,900,1000,1100, \ldots\}$. The acc improvement on $n_{4}$ vs. $n_{3}, n_{5}$ vs. $n_{4}$, and $n_{6}$ vs. $n_{5}$ are all less than $0.3 \%$, while the computing costs significantly grow with the increase of $n$. So sampling process is terminated. Among the latter six committees, the committee on $n_{3}$ is adopted as the final classifier for prediction due to its good performance. Additionally, to depict the time vs. $n$ curve, we add two excess sample points 700 and 1500 which are all marked with symbol *.

Table 2. Time and Accuracy on Different Sample Sizes for the Credit Card Database

\begin{tabular}{lclll}
\hline $\mathrm{N}$ & Time $(\mathrm{s})$ & acc1 $(\%)$ & acc2 $(\%)$ & acc $(\%)$ \\
\hline 200 & 1 & 69.09 & 82.57 & 69.7 \\
\hline 400 & 10 & 71.52 & 81.14 & 73.2 \\
\hline 600 & 60 & 72.00 & 80.00 & 73.4 \\
\hline 800 & 210 & 71.52 & 84.00 & 73.7 \\
\hline 900 & 390 & 70.06 & 84.57 & 73.6 \\
\hline 1000 & 660 & 71.64 & 81.71 & 73.4 \\
\hline 1100 & 1200 & 70.40 & 85.65 & 73.1 \\
\hline $700^{*}$ & 120 & 71.39 & 84.00 & 73.6 \\
\hline $1500^{*}$ & 4800 & 71.89 & 83.51 & 73.8 \\
\hline
\end{tabular}

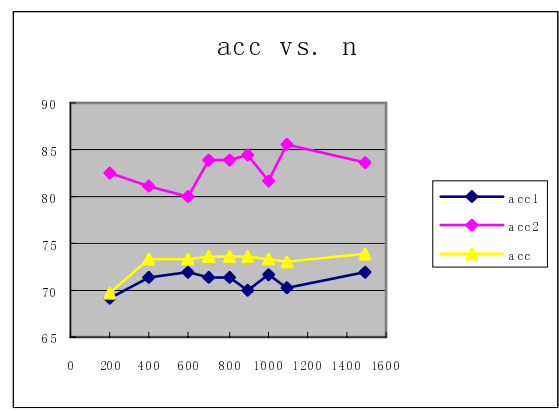

Fig. 2. Result of acc vs. $\mathrm{n}$ for Credit Card Database

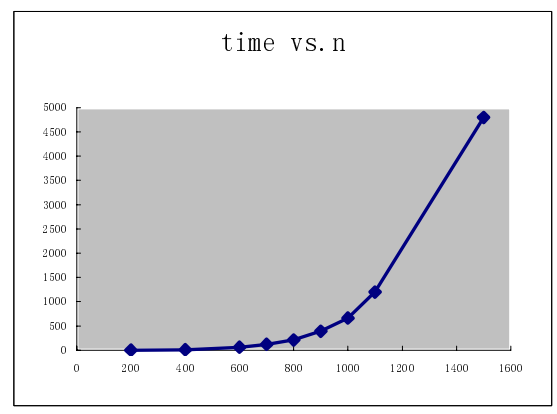

Fig. 3. Result of time vs. $n$ for Credit Card Database 
Similarly, we have following results with respect to the census database. The approximately optimal sample size is $n_{6}=1700$.

Table 3. Time and Accuracy on Different Sample Sizes for the Census Database

\begin{tabular}{ccccc}
\hline $\mathrm{N}$ & time $(\mathrm{s})$ & Acc1 $(\%)$ & Acc $(\%)$ & Acc $(\%)$ \\
\hline 300 & 8 & 78.52 & 85.20 & 80.89 \\
\hline 600 & 9 & 78.07 & 86.00 & 80.88 \\
\hline 900 & 25 & 77.89 & 87.42 & 81.27 \\
\hline 1200 & 55 & 78.44 & 87.25 & 81.57 \\
\hline 1500 & 143 & 78.42 & 86.38 & 81.25 \\
\hline 1600 & 200 & 78.57 & 86.72 & 81.47 \\
\hline 1700 & 230 & 78.77 & 86.69 & 81.58 \\
\hline 1800 & 290 & 78.13 & 87.75 & 81.54 \\
\hline $1900^{*}$ & 340 & 78.79 & 86.77 & 81.62 \\
\hline $2500^{*}$ & 940 & 78.56 & 86.86 & 81.51 \\
\hline
\end{tabular}

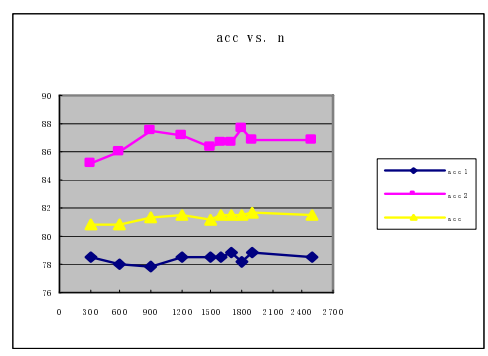

Fig. 4. Result of acc vs. $\mathrm{n}$ for the census database

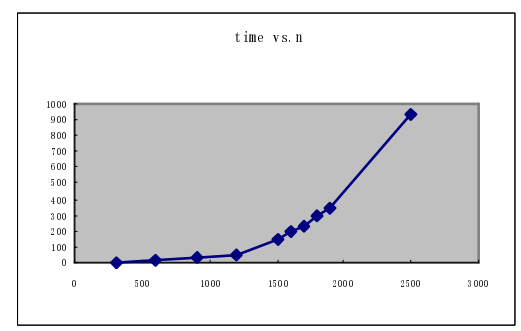

Fig. 5. Result of time vs. $n$ for the census database

\subsection{Analysis of Results}

From the results of two databases, we have three empirical results. Firstly, the Dynamic Committee-Classifier Scheme on MCLP can effectively find the approximately optimal example size for MCLP classification method with respect to a given dataset, i.e., the approximately optimal example size really exists. Considering the time cost, 800 is enough for the first dataset, which only accounts for $20 \%$ of original training dataset; 1700 is sufficient for the second dataset, which only equals to $3.5 \%$ of the whole training set. Secondly, it can efficiently find the approximately optimal sample size. For example, in the credit card database, we can see $4800>1+10+60+210+390+660+1200$. That is to say, if we sample a sample with 1500 cases, its computing cost outclasses the whole computing time of scheme $\{200,400,600,800,900,1000,1100\}$, while its accuracy rate only has 0.1 percent of improvement. From the time vs. $n$ curve, we can also see that time cost is unacceptable when $\mathrm{n}$ is too large. Lastly, we find some laws reflecting the relationship between acc and $n$, between time and $n$, and between number of attributes and time. The time vs. $n$ curve is in the form of power function. 


\section{Conclusions and Future Efforts}

To enhance computation efficiency, sampling is an important strategy in MCLP classification. To ensure the credibility and efficiency of sampling, we integrate progressive sampling and classification committee, and then design a dynamic classification committee scheme for MCLP classification method. The experimental results have shown that our idea is feasible and the scheme is effective and efficient in exploring an approximately optimal sample size. The empirical results also help us to further investigate some general specialities of MCLP. From the above two datasets, we can see the time vs. $n$ curve is in the form of power function. But we can't perfectly depict the form of $a c c$ vs. $n$ curve due to deficiency of number of samples. In our future researches, we will further explore more general forms of functions $a c c=f(n)$ and time $=f(n)$.

\section{References}

1. Shi, Y., Wise, M., Luo, M., Lin, Y.: Data mining in credit card portfolio management: a multiple criteria decision making approach. In: Koksalan, M., Zionts, S. (eds.): Multiple Criteria Decision Making in the New Millennium, Springer, Berlin(2001)427-436

2. Shi, Y., Yu, P. L.: Goal setting and compromise solutions. In: Karpak, B., Zionts, S. (eds.): Multiple Criteria Decision Making and Risk Analysis Using Microcomputers, SpringerVerlag, Berlin (1989) 165-204

3. Shi,Y.: Multiple Criteria Multiple Constraint-levels Linear Programming: Concepts, Techniques and Applications. World Scientific Publishing, River Edge, New Jersey (2001)

4. Shi, Y., Peng, Y., Xu, W. ,Tang, X.: Data Mining via Multiple Criteria Linear Programming: Applications in Credit Card Portfolio Management. International Journal of Information Technology and Decision Making. 1 (2002) 131-151.

5. Kou, G., Liu, X., Peng, Y., Shi, Y., Wise, M., Xu, W.: Multiple Criteria Linear Programming to Data Mining: Models, Algorithm Designs and Software Developments. Optimization Methods and Software. Vol. 18(2003) 453-473

6. John, G., Langley, P.: Static Versus Dynamic Sampling for Data Mining. In : Simoudis, E., Han, J.W., Usama M. Fayya( eds): Proceedings of the Second International Conference on Knowledge Discovery in Databases and Data Mining, AAAI/MIT Press( 1996)367-370

7. Provost, F., Jensen, D., Oates, T.: Efficient progressive sampling. In: Proceedings of the Fifth KDDM, ACM Press, New York(1999) 23-32

8. Breiman, L.: Bagging predictors. Machine Learning. 24(2)(1996)123-140

9. Freund. Y., Schapire, RE. :Experiments with a new boosting algorithm. In: Proceedings of 13th International Conference on Machine Learning (1996) 148-156

10. Ho, T.K.: The random subspace method for constructing decision forests. IEEE transactions on Pattern Analysis and Machine Intelligence.20(8)(1998)832-844

11. Breiman, L.: Random forests. Machine Learning. 45(1)( 2001)5-32

12. Freund, Y., Schapire, RE.: Discussion of the paper Arcing Classifiers by Leo Breiman. The Annals of Statistics 26 (3) (1998) 824-832

13. Efron, B., Tibshirani, R.: An Introduction to the Bootstrap. Chapman\& Hall, New York(1993) 